\title{
Solving hybrid-vehicle routing problem using modified simulated annealing
}

\author{
Nour Alsumairat, Mahmoud Alrefaei \\ Department of Mathematics and Statistics, Jordan University of Science and Technology, Irbid, Jordan
}

\begin{tabular}{l} 
Article Info \\
\hline Article history: \\
Received Jan 1, 2021 \\
Revised May 18, 2021 \\
Accepted Jun 1, 2021 \\
\hline
\end{tabular}

\section{Keywords:}

Hybrid vehicle

Routing problem

Simulated annealing

Transportation

\begin{abstract}
In this paper, we consider the hybrid vehicle routing problem (HVRP) at which the vehicle consumes two types of power: fuel and electricity. The aim of this problem is to minimize the total cost of travelling between customers, provided that each customer is visited only once. The vehicle departs from the depot and returns after completing the whole route. This optimization problem is solved using a modified simulated annealing (SA) heuristic procedure with constant temperature. This approach is implemented on a numerical example and the results are compared with the SA algorithm with decreasing temperature. The obtained results show that using the SA with constant temperature overrides the SA with decreasing temperature. The results indicate that SA with decreasing temperature needs twice the number of iterations needed by the SA with constant temperature to reach a near optimum solution.
\end{abstract}

This is an open access article under the CC BY-SA license.

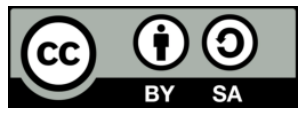

\section{Corresponding Author:}

Mahmoud Alrefaei

Department of Mathematics and Statistics

Jordan University of Science and Technology

Irbid 22110, Jordan

Email: alrefaei@just.edu.jo

\section{INTRODUCTION}

Vehicle routing problem (VRP) was first introduced by Dantzig [1], where the optimum routing was considered for a fleet of gasoline-delivery trucks traveling between a depot and terminal customers and some service stations. They proposed the first mathematical linear programming formulation and algorithmic approach. Cao [2] listed eight points to classify the VRP according to; number of distribution centers, type of vehicle, characteristics of the task, time constraints, vehicle loading, optimization of the number of goals, ownership of the points, and mastering the information of certainty.

Vehicles capacity plays a very big role in the VRP; therefore, researchers add the capacity constraints to the original problem to align with real world case which is known as capacitated vehicle routing problem (CVRP). For example, Faulin et al. [3] have used algorithms with environmental criteria to solve the CVRP, where environmental costs were considered together with cost, distance, number of vehicles and profit. Mahvash et al. [4] proposed the CVRP with new constraints for three-dimensional shaped products, where they have used a column generation (CG) technique-based heuristic.

On the other hand, in vehicle routing problem with time windows (VRPTW); the service time at each customer is within a specific time window, a two stage algorithm is proposed by Lim [5] to solve VRPTW; in the first stage the algorithm minimizes the number of vehicles with an ejection pool, then it minimizes the total travel distance using a multi-start iterated hill-climbing algorithm. Yuliza et al. [6] solved an open capacitated vehicle routing problem for waste transporting problems, with uncertain amount of waste and travel time. 
VRPs can be solved using different approaches, including the exact approach; El-Sherbeny [7] classified the exact method for the VRPTW into three categories: Lagrange relaxation-based methods, column generation, and dynamic programming. Iori et al. [8] proposed an exact approach based on a branchand-cut algorithm for the capacitated VRP. The problem was considered with two-dimensional additional loading constraints. Baldacci et al. [9] used an exact method that decomposes the two-echelon capacitated vehicle routing problems (2E-CVRP) into a limited set of multi depot capacitated vehicle routing problems with side constraints.

Heuristic algorithms have been used to replace the decision criteria of the exact approach algorithm to effectively narrow the search space which is known as intelligent heuristic algorithm. For example, Tavakoli [10] used particle swarm optimization (PSO) to solve the CVRP, they referred to each found solution as a point in n-dimensional space that has an initial velocity, while particles (solutions) were evaluated according to some fitness criteria. However, PSO might be stuck into local optima; therefore, multi-swarm particle swarm optimization (MSPSO) has been used by other researchers, where the swarm is divided into sub-swarms, and the movement process of a particle is enhanced with a probability of bisection method, see Alfarisy et al. [11]. Puspita et al. [12], [13] have modeled an optimization waste transport as a CVRP and solved it using branch and bound method with the aid of LINGO 13.0.

One of the most important methods that can be used for solving VRP is simulated annealing which is a common local search meta-heuristic used to address discrete, and to a lesser extent, continuous optimization problems. Annealing is referred to, as tempering certain alloys of metal, glass, or crystal by heating above its melting point, holding its temperature, and then cooling it very slowly until it solidifies into a perfect crystalline structure. The simulation of this process is known as simulated annealing (SA), the basic idea is to find a global minimum amount of energy to produce a defect-free crystal material. The first appearance of simulated annealing was in 1953 by Metropolis et al. [14]. In Kirkpatrick et al. [15] developed the similarities between the statistical mechanics and combinatorial optimization. The key algorithmic feature of simulated annealing is that it provides a mean to escape local optimum by allowing hill-climbing moves (i.e., moves which worsen the objective function value), as the temperature parameter decreases, the probability of accepting a worse objective function will also decrease.

The SA algorithm starts with an initial solution, Szabo [16] tested the effectiveness of choosing the initial solution in SA for a flow shop problem. Then, the objective function is evaluated, after that a new (neighborhood) solution is generated, Cruz-Chavez [17] presented a mechanism to generate a new neighborhood solution for the job shop scheduling problem (JSSP). Initial temperature plays an important role in SA since the acceptance ratio strongly depends on the temperature, Shakouri et al. [18] discussed the initial temperature and proposed an approach to speed up the algorithm of SA while obtaining accurate solutions for the traveling salesman problem (TSP). The generated solution is compared to the old solution, if the new solution has better objective function value, the algorithm moves to that solution, otherwise a random number between 0 and 1 is generated and compared with a probability ratio that decides whether to accept or reject the new solution. Baizal et al. [19] used the simulated annealing for solving the TSP and have shown through examples that the SA much better than some other competent methods. Another version of simulated annealing that uses constant temperature was presented by Alrefaei [20] and another version uses the ranking and selection method was proposed by Alrefaei [21]. Ariyani [22] solved the VRPTW using a hybrid GA-SA algorithm. Yu et al. [23] have used the simulated annealing to solve the hybrid vehicle routing problem (HVRP). The HVRP is an extension of the original VRP with the addition of the ability to recharge the vehicle electrically. More applications of simulated annealing on multi objective optimization can be found in [24]-[29]. More recent applications of the simulated annealing algorithm for solving engineering problems can be found in [30]-[33].

The advantage of simulated annealing algorithm is that it reduces the number of solutions that must be tested to find the optimum solution. Moreover, the structure of the SA algorithm is consistent compared with other heuristic algorithms. Annealing at constant and decreasing temperatures were used in this study. The use of constant temperature considered here gives the algorithm more freedom to move around the state space and rapidly finds the optimal solution, Alrefaei and Andradottir [20]. This paper is organized as follow; in section 2, we formulate the VRP problem, in section 3, we present the methodology used to solve the problem, in section 4, a numerical example is given, and in section 5, we include the results followed by concluding remarks.

\section{RESEARCH METHOD}

Vehicle routing problem (VRP) is usually solved using the transport route optimization. The main goal of the vehicle routing optimization is to minimize the total cost with offering the needed service for every customer. This study employs parameters based on previous research, Yu et al. [29]. 
The following notations are needed throughout this paper:

$v_{0} \quad$ : Depot index.

$V \quad:$ The set of all customers.

El : The set of electric stations.

$\mathrm{Fu} \quad$ : The set of fuel stations.

Ref : The set of all electric and fuel stations together with the depot.

A : The set of all stations and all customers together with the depot.

De : The remaining distance that the vehicle can cover using electricity.

Df : The remaining distance that the vehicle can cover using fuel.

$T_{\text {max }} \quad$ : Maximum time that the vehicle is not allowed to exceed per round.

$x_{\mathrm{i}, \mathrm{j}} \quad$ : Binary variable equal to one if the vehicle traveled from vertex $i$ to vertex $j$ and zero, otherwise.

$\tau_{\mathrm{i}, \mathrm{j}} \quad:$ The time needed to travel form vertex $i$ to vertex $j$.

$R e_{i, j} \quad:$ The distance from vertex $i$ to vertex $j$ that is covered using electricity.

$R f_{\mathrm{i}, \mathrm{j}} \quad:$ The distance from vertex $i$ to vertex $j$ that is covered using fuel.

Re : The maximum distance that can be traveled when the vehicle is electrically fully charged.

$R f \quad:$ The maximum distance that can be traveled when the vehicle is fully filled with fuel.

$E c_{\mathrm{i}, \mathrm{j}} \quad$ : The electricity cost to travel from vertex $i$ to vertex $j$.

$F c_{\mathrm{i}, \mathrm{j}} \quad:$ The fuel cost to travel from vertex $i$ to vertex $j$.

$D_{\mathrm{i}, \mathrm{j}} \quad:$ The distance between vertex $i$ and vertex $j$.

$E c_{r} \quad$ : The electricity consumption rate.

Ec : The electricity cost per kwh.

$F c_{r} \quad$ : The fuel consumption rate.

FC : The fuel cost per gallon.

The HVRP is defined as a directed graph $G=(A, E)$, where the vertex set A is a combination of the depot $v_{0}$, the electric stations set, the fuel stations set, and the customers set. The set Ref is the depot together with the electric stations and fuel stations. The set of edges $E=\{(i, j): i, j$ is the index for the visited customer or station\}. Each pair in $E$ is associated with travel time, distance, and total cost till that point. As an example, denote the depot by $v_{0}$, the electric stations set by $E l=\left\{E_{1}, E_{2}\right\}$, the fuel stations set by $F u=\left\{F_{3}, F_{4}\right\}$ and the customers set by $V=\left\{v_{5}, v_{6}, v_{7}, v_{8}, v_{9}, v_{10}, v_{11}, v_{12}\right\}$, then the set $\mathrm{A}=$ $\left\{v_{0}, E_{1}, E_{2}, F_{3}, F_{4}, v_{5}, v_{6}, v_{7}, v_{8}, v_{9}, v_{10}, v_{11}, v_{12}\right\} \quad$ and $E=\{(i, j): i, j \in A, j \neq \mathrm{i}\}$. The mathematical formulation of HVRP is as (1):

$$
\min \sum_{\mathrm{i}, \mathrm{j} \in \mathrm{A}}\left(\mathrm{Ec}_{\mathrm{i}, \mathrm{j}}+\mathrm{Fc}_{\mathrm{i}, \mathrm{j}}\right)
$$

Subject to

$$
\begin{aligned}
& \sum_{\substack{\mathrm{j} \in \mathrm{A} \\
\mathrm{j} \neq \mathrm{i}}} \mathrm{x}_{\mathrm{i}, \mathrm{j}}=1, \forall \mathrm{i} \in \mathrm{V} \\
& 0<\sum_{i, j \in A} \mathrm{x}_{\mathrm{i}, \mathrm{j}} \tau_{i, j}<T_{\max } \\
& 0<\mathrm{Rf}_{\mathrm{i}, \mathrm{j}}+\mathrm{Re}_{\mathrm{i}, \mathrm{j}} \leq \mathrm{Re}+\mathrm{Rf}, \quad \forall i, j \in \mathrm{A} \\
& E c_{i, j}=D_{i, j} * E c_{r} * E c \\
& F c_{i, j}=D_{i, j} * F c_{r} * F C
\end{aligned}
$$

The objective function is given in (1) which is the total cost including electricity cost as well as fuel cost that can be evaluated using formulas (5) and (6). The first constraint is to make sure that all customers were visited only once is given by (2). The second constraint is to keep the total time for every round less than the maximum time that the vehicle is not allowed to exceed (3). The third constraint is to ensures that any movement from vertex $i$ to vertex $j$ can be covered at most by the electricity and fuel storage (4). The vehicle can be recharged at the depot as well as the electric stations. The vehicle can be refueled at the depot as well as fuel stations.

Hybrid vehicle routing problem (HVRP) is an extension of the green vehicle routing problem (GVRP). In GVRP, the vehicle is pure electric. However, in HVRP, the vehicle runs on electricity and fuel which extends the distance travelled. Therefore, hybrid vehicle can serve more customers. Mathematically, 
the problem is to minimize the total cost $T_{c}$ over the set of feasible solutions S. Hybrid vehicle can use both electric and fuel storage, in our study, we give the priority to consume electric, since it costs less according to the used vehicle specifications. Figure 1 shows an example of HVRP.

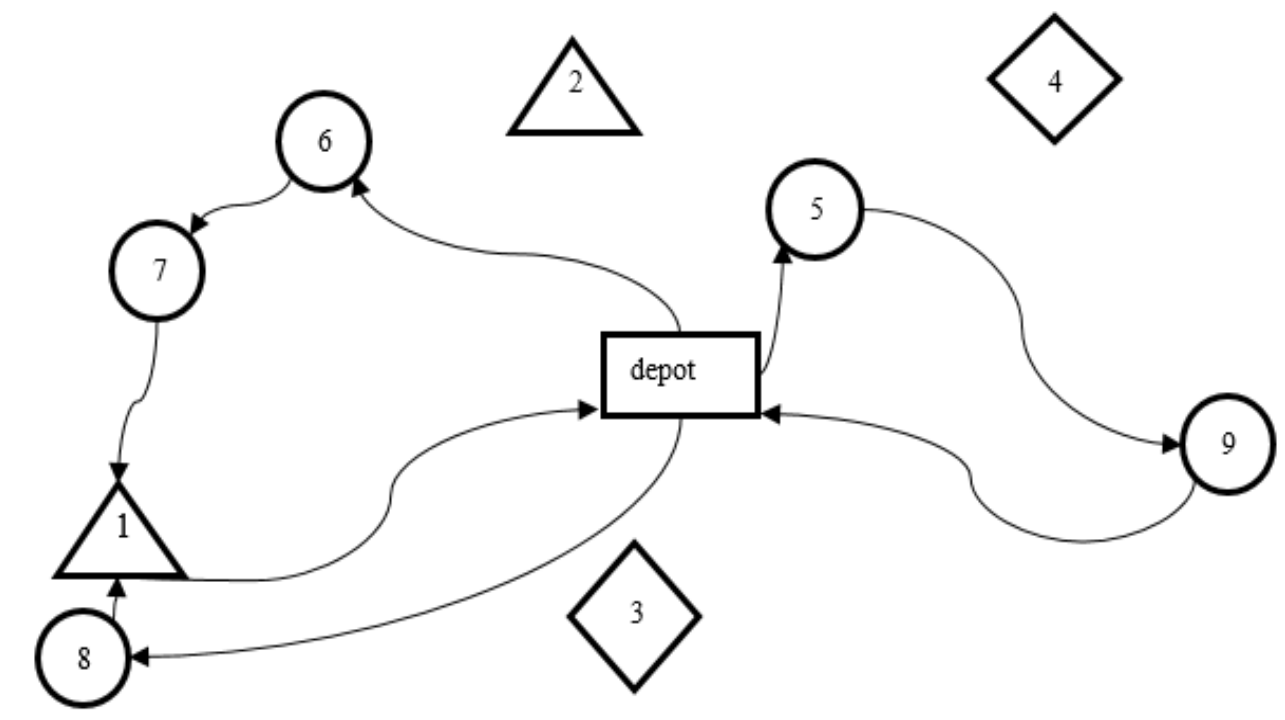

\section{Depot $\square$ Customers $\bigcirc$ Electric Stations $\triangle$ Fuel Stations}

Figure 1. Example of a vehicle need three rounds to complete the trip

In HVRP, the vehicle departs from the depot and serves as many customers as it can. At each step, electricity and fuel levels, remaining time to reach next costumer, and closeness to electricity or fuel recharge station need to be checked. If the electricity or fuel level is not enough to reach the nearest customer; a check on the nearest station need to be made. If the electricity and fuel levels are not enough to reach a recharge station, the vehicle goes back to the depot, refills fuel and electricity, and starts a new round. The trip ends when all customers are served. There are no constraints on the number of rounds (departure from and return to the depot is considered as one round).

To solve the HVRP, a modified version of the simulated annealing technique with constant temperature is used to find the optimum solution. Many local optimizers can be handled using this method since it has the hill climbing feature as the original simulated annealing algorithm. At the beginning, a feasible initial solution $x$ for the problem is used, then, the objective function (total travel cost) $f(x)$ is computed based on the initial solution, after that, a candidate solution $y$ is generated based on replacing one customer with another one randomly. Then, the total travel cost $f(y)$ is computed at $y$, if $f(y)<f(x)$, then the algorithm accepts the move to the candidate solution. However, if $f(y) \geq f(x)$, then there is possibility that a better solution is hidden behind the candidate solution, so the candidate solution $y$ will be accepted with probability $\operatorname{Prob}(x, y)$ that depends on the difference between the two objective function values $\Delta=[f(y)-f(x)]$. The acceptance probability $\operatorname{Prob}(x, y)$ is given by:

$$
\operatorname{Prob}(x, y)= \begin{cases}e^{-\left(\frac{\Delta}{T}\right)} & \text { if } f(y) \geq f(x) \\ 1 & \text { other wise }\end{cases}
$$

where $T$ is a controller parameter; called the temperature. In implementation, a uniform random number $U$ between 0 and 1 is generated and compared with $\operatorname{Prob}(x, y)$, if $U \leq \operatorname{Prob}(x, y)$, the move is accepted, otherwise a new solution is generated from the neighborhood of $x$, and the process is repeated until a stopping rule is reached. The simulated annealing procedure is presented in the flow chart in Figure 2. 


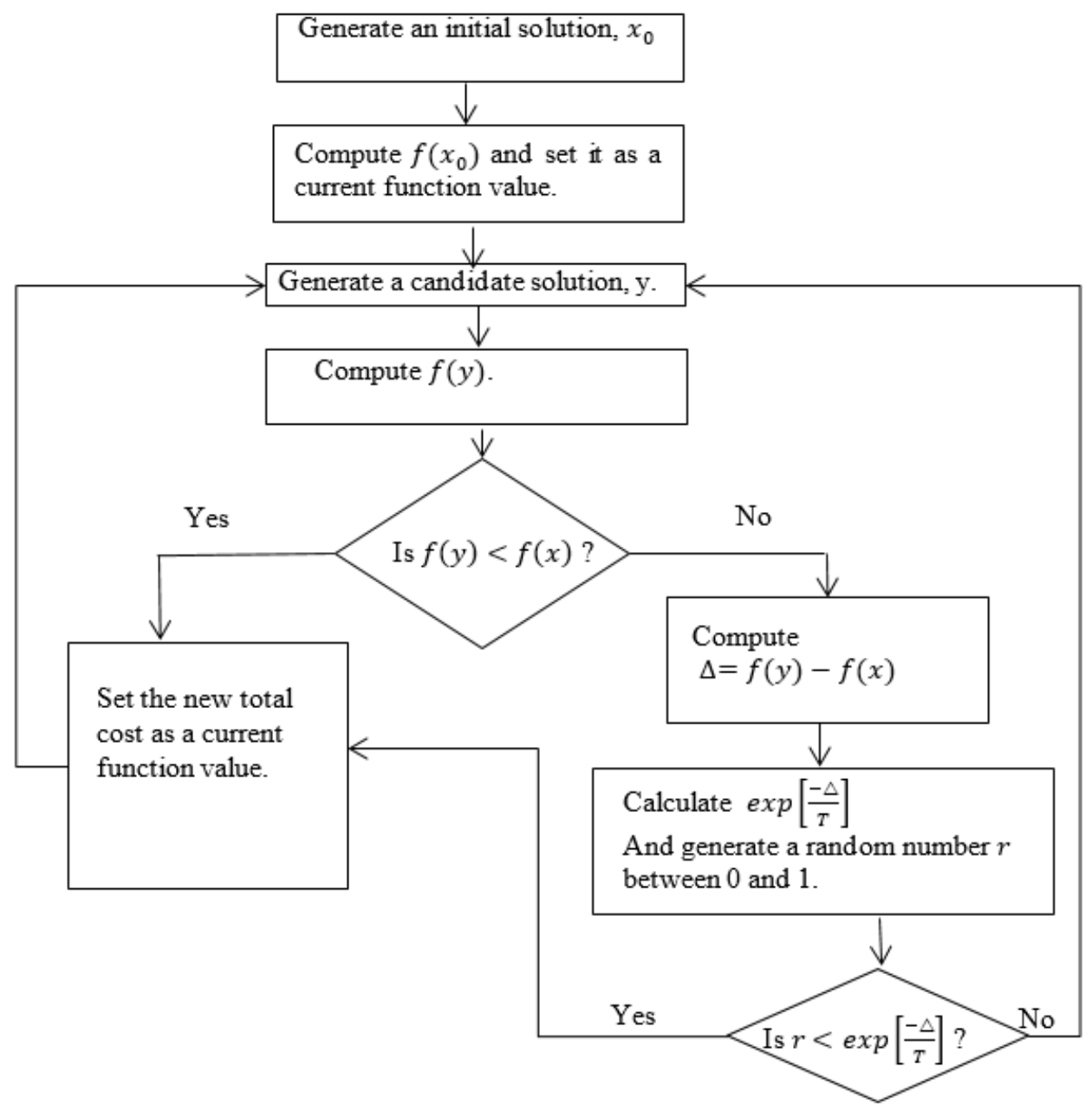

Figure 2. Simulated annealing procedure with constant temperature

\section{NUMERICAL EXAMPLE}

Consider the HVRP problem with depot $v_{0}$, two electric stations $\left\{E_{1}, E_{2}\right\}$, two fuel stations $\left\{F_{3}, F_{4}\right\}$, and seven customers $\left\{c_{5}, c_{6}, c_{7}, c_{8}, c_{9}, c_{10}, c_{11}, c_{12}\right\}$. The vehicle specifications are given in Table 1 . The vehicle should visit all customers, starting from the depot and return to it, the HVRP described in (1)-(6) is solved using SA algorithm. The SA is implemented using constant temperature and is compared with a decreasing temperature as suggested by [9]. We use three different values for the constant temperature $T=1, T=5$ and $T=10$. We use the solution that has the minimum value so far as the optimal solution as in the algorithm of Alrefaei and Andradóttir [20]. The candidate solution is generated by replacing a random customer with another random one. We used two types of stopping rules, in the first stopping rule, the algorithm stops when there is no improvement for five consecutive iterations, while in the second stopping rule, we ran a specific number of iterations, then stop the algorithm. Now, we discuss the results in detail. The distances between customers, stations, and depot are shown in Table 2.

Table 1. Vehicle specifications

\begin{tabular}{cc}
\hline Specification & \\
\hline Electric consumption rate & $0.5 \mathrm{KWh} / \mathrm{mile}$ \\
Maximum electric distance & $21 \mathrm{miles}$ \\
Electric cost & $\mathrm{US} \$ 0.12 / \mathrm{KWh}$ \\
Fuel consumption rate & $17.7 \mathrm{mpg}$ \\
Maximum fuel distance & $150.5 \mathrm{miles}$ \\
Fuel cost & $\mathrm{US} \$ 4.18 / \mathrm{gal}$ \\
Maximum round time & 11 hours \\
Speed Limit & $40 \mathrm{mph}$ \\
\hline
\end{tabular}


Table 2. Distance matrix

\begin{tabular}{|c|c|c|c|c|c|c|c|c|c|c|c|c|c|}
\hline & 0 & Es1 & Es2 & Fs1 & Fs2 & $\mathrm{C} 5$ & C6 & $\mathrm{C} 7$ & $\mathrm{C} 8$ & C9 & $\mathrm{C} 10$ & $\mathrm{C} 11$ & $\mathrm{C} 12$ \\
\hline 0 & 0.00 & 26.71 & 14.34 & 95.78 & 196.87 & 49.23 & 18.4 & 22.37 & 46.17 & 66.9 & 33.00 & 79.00 & 55.00 \\
\hline Es2 & 14.34 & 123.46 & 0.00 & 47.65 & 72.77 & 8.19 & 110.02 & 27.15 & 31.7 & 104.31 & 45.00 & 43.00 & 43.00 \\
\hline Fs 2 & 196.87 & 184.99 & 72.77 & 115.06 & 0.00 & 69.69 & 172.85 & 77.38 & 41.46 & 41.28 & 74.00 & 48.00 & 50.00 \\
\hline $\mathrm{C} 5$ & 49.23 & 130.96 & 8.19 & 54.9 & 69.69 & 0.00 & 117.43 & 34.72 & 18.25 & 12.37 & 54.00 & 23.00 & 22.00 \\
\hline C6 & 18.4 & 13.83 & 110.02 & 62.55 & 172.85 & 117.43 & 0.00 & 95.66 & 137.92 & 67.79 & 70.00 & 103.00 & 93.00 \\
\hline C9 & 66.9 & 71.15 & 104.31 & 32.74 & 41.28 & 12.37 & 67.79 & 78.41 & 119.48 & 0.00 & 84.00 & 25.00 & 18.00 \\
\hline $\mathrm{C} 10$ & 33.00 & 60.00 & 45.00 & 33.00 & 74.00 & 54.00 & 70.00 & 36.00 & 91.00 & 84.00 & 0.00 & 57.00 & 50.00 \\
\hline C11 & 79.00 & 80.00 & 43.00 & 56.00 & 48.00 & 23.00 & 103.00 & 32.00 & 63.00 & 25.00 & 57.00 & 0.00 & 12.00 \\
\hline $\mathrm{C} 12$ & 55.00 & 92.00 & 43.00 & 86.00 & 50.00 & 22.00 & 93.00 & 29.00 & 53.00 & 18.00 & 50.00 & 12.00 & 0.00 \\
\hline
\end{tabular}

\section{RESULTS AND DISCUSSION}

To find the optimum solution, we first generate an initial solution. Starting from the depot, check the ability to serve the nearest customer by checking the time as well as the electricity and fuel storage. Find the nearest customer and check if it can be reached and check the nearest station to the nearest customer, if it cannot be reached, then move to the nearest station of the current node. If the time is not enough to reach any of the mentioned nodes it will return to the depot and starts a new round. If the remaining time or power is not enough to return to the depot, the algorithm will reveal an infeasible solution. The total cost will be calculated based on the distance travelled, the power (electricity and fuel) consumption rate, and the power cost.

Table 3 shows the generated initial solution for visiting all customers where the final total cost is \$94.58. In this solution, the vehicle visited one electric station, which has index 2, and one fuel station, which has index 3. The algorithm starts from customer 6 then visits customer 9 then 5 and then 8 . After that, the vehicle is run out of enough electricity and fuel, therefore the algorithm looks for the nearest station, which is node 3 , after refueling, the vehicle moves to customer 10 then customer 7 and then customer 12 , then it needs to recharge again from station 2, and since it couldn't go to other customer because the remaining time is not enough, it returns to the depot to start a new round to continue serving the rest customers. Then a candidate solution is generated, by changing the order of customers to be served and the total cost is computed. After that, we compare the current value of the total cost with the new one. We first consider a constant temperature with three different values for $T, T=1,5$ and 10 and then use a decreasing temperature $T_{0}=20, T_{i+1}=0.8 * T_{i}$. The four cases are described below.

\subsection{The case when $T=1$}

Figure 3 shows the SA performance when $T=1$. Where the dotted line (red) shows the objective function of the candidate solutions and the strict line shows the current objective function of the current solutions. If the strict line moves upward, then a worse solution is accepted, this is because a better solution may be hidden behind this solution. Note that the probability of accepting any worse objective function value is very small, as shown in Figure 3, it almost does not accept any worse function value, this means that algorithm stuck in a local minimum solution. It needs 60 iterations to reach the optimum value, which indicates that it keeps performing good but approaches the optimum slowly. According to the first stopping rule which is to have five iterations without improvement in the objective function value; the optimum value was $\$ 63.94$ resulted after 12 iterations, which is a good solution, but is not the minimum. The minimum was obtained at iteration 65 with a value of $\$ 59.11$.

Table 3. The initial solution

\begin{tabular}{ccccc}
\hline From & To & Electricity cost & Fuel Cost & Total Cost \\
\hline 0 & 6 & $E c=0.55$ & $F c=0.00$ & $T c=0.55$ \\
6 & 9 & $E c=0.09$ & $F c=15.25$ & $T c=15.89$ \\
9 & 5 & $E c=0.00$ & $F c=2.89$ & $T c=18.78$ \\
5 & 8 & $E c=0.00$ & $F c=4.27$ & $T c=23.05$ \\
8 & 3 & $E c=0.00$ & $F c=5.39$ & $T c=28.44$ \\
3 & 10 & $E c=0.00$ & $F c=7.72$ & $T c=36.17$ \\
10 & 7 & $E c=0.00$ & $F c=8.42$ & $T c=44.59$ \\
7 & 12 & $E c=0.00$ & $F c=6.78$ & $T c=51.38$ \\
12 & 2 & $E c=0.00$ & $F c=10.06$ & $T c=61.45$ \\
2 & 0 & $E c=0.43$ & $F c=10.06$ & $T c=61.88$ \\
0 & 11 & $E c=0.63$ & $F c=13.57$ & $T c=76.09$ \\
11 & 0 & $E c=0.00$ & $F c=18.49$ & $T c=94.58$ \\
\hline
\end{tabular}




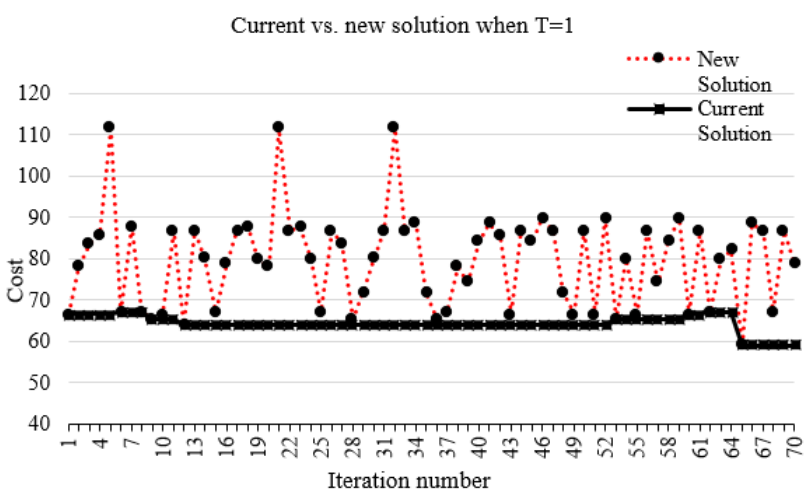

Figure 3. Current versus new solution when $\mathrm{T}=1$

\subsection{The case when $T=5$}

Now we apply the SA algorithm with a constant temperature value $\mathrm{T}=5$. In this case, the probability of accepting any worse objective function value is still small but it is better than the case where $\mathrm{T}=1$. The results are depicted in Figure 4.

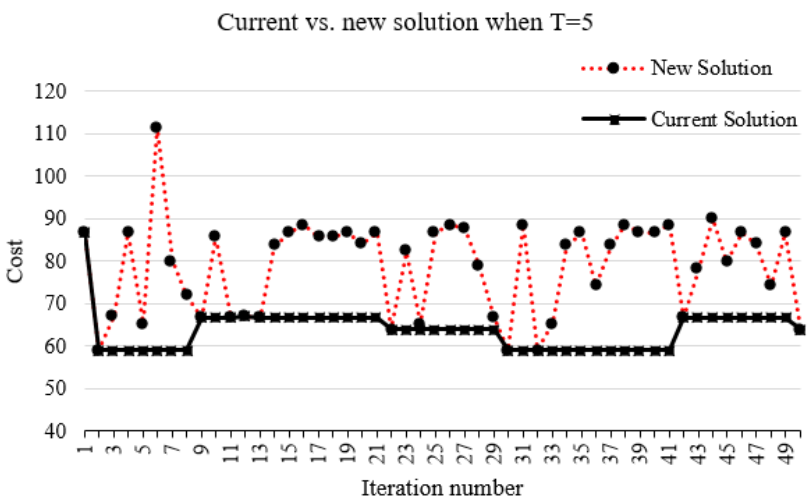

Figure 4. Current versus new solution when $\mathrm{T}=5$

\subsection{The case when $T=10$}

The SA algorithm with a constant value $\mathrm{T}=10$. In this case, the probability of accepting any worse objective function value is larger than the previous two cases. Therefore, the current solution varies for all iterations, and it reached the minimum $(\$ 59.11)$ at iteration 12, as shown in Figure 5.

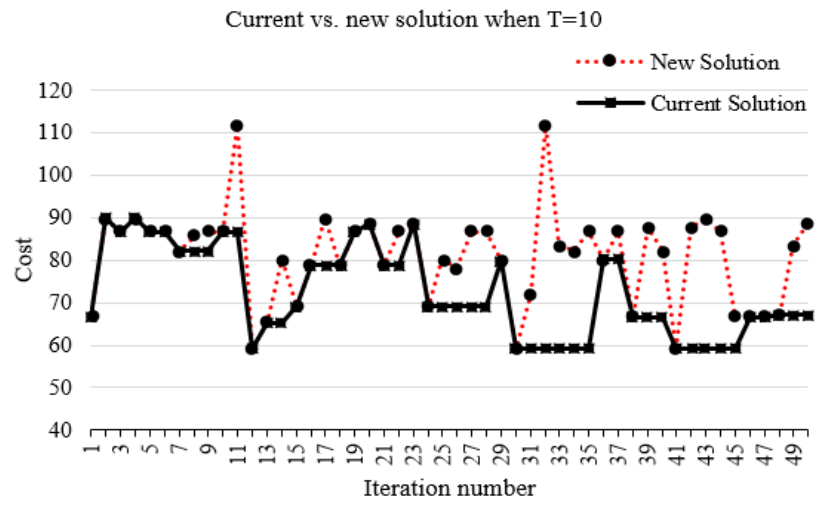

Figure 5. Current versus new solution when $\mathrm{T}=10$ 


\subsection{The case when $T_{0}=20$ and $T_{i+1}=0.8 * T_{i}$}

Now to compare the results In the SA algorithm with a decreasing value starting at $\mathrm{T}=20$ and decreases with ratio of 0.8 . The algorithm accepts worse function value with high probability at the beginning, however, it did not accept any worse value for the objective function from iteration 9 to iteration 29. It reached the minimum (\$59.11) at iteration 22, as shown in Figure 6.

Figure 7 presents the average performance of the algorithm over 10 replications using the above four cases of the temperature $T$. It is clear that, using a constant temperature $T=10$, allows the algorithm to explore the state space freely and then locate the optimal solution faster. However, using a small temperature $T=1$ or $T=5$, the algorithm does not move freely so it stick in a local optimal solution. Moreover, using a decreasing temperature ends in locating a local optimal solution also, which requires restarting the algorithm to explore the state space. Table 4 shows a comparison between the optimum function value for the four cases of the temperature value using the two stopping rules over 10 replications.

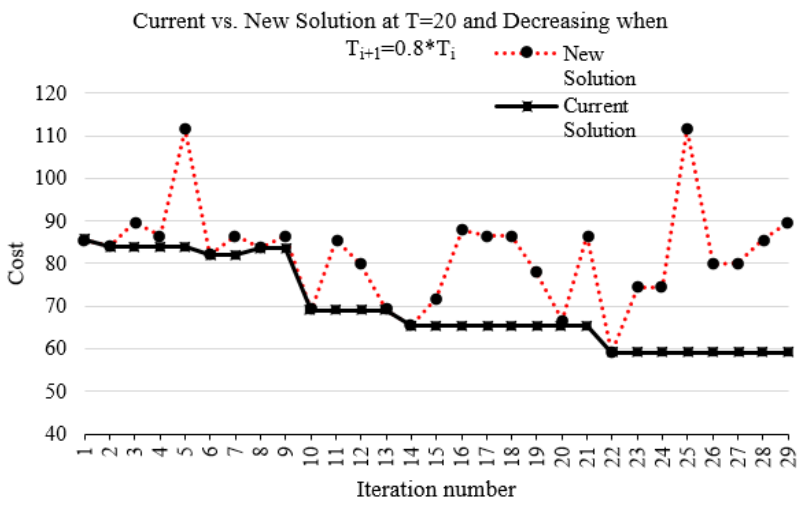

Figure 6. Current versus new solution at $\mathrm{T}=20$ and deceasing at $T_{i+1}=0.8 * T_{i}$

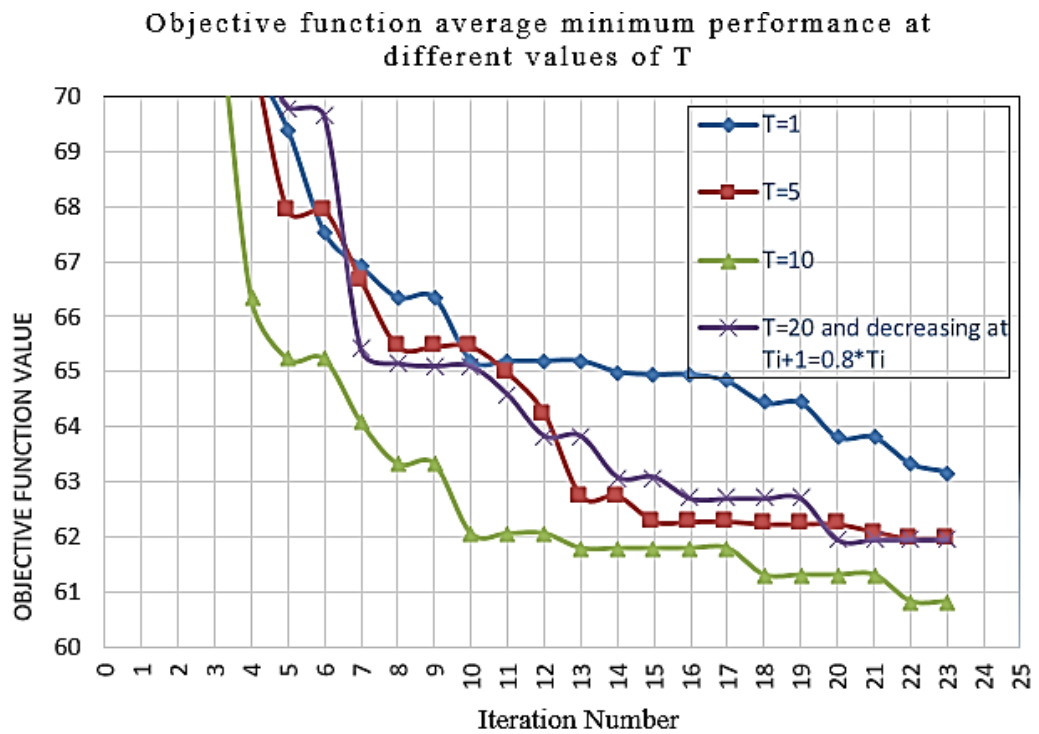

Figure 7. The average performance of the SA over 10 replications using four different values of the temperature

Table 4. A comparison between the four cases of the temperature value

\begin{tabular}{cccc}
\hline Temperature value & The first stopping rule & The second stopping rule & Average number of iterations \\
\hline $\mathrm{T}=1$ & 66.343 & 63.178 & 23 \\
$\mathrm{~T}=5$ & 64.959 & 61.945 & 22 \\
$\mathrm{~T}=10$ & 62.809 & 60.826 & 22 \\
$T_{i+1}=0.8 * T_{i}, T_{0}=20$ & 65.339 & 61.945 & 19 \\
\hline
\end{tabular}




\section{CONCLUSION}

In this paper, we have studied the hybrid vehicle routing problem (HVRP) at which a vehicle is planned to visit all customers only once and return to the depot, the simulated annealing algorithm with constant temperature was used to solve the HVRP, this allows the algorithm to explore the state space freely, especially for large values of $\mathrm{T}$. The results of the proposed method are compared with the SA algorithm using decreasing temperature. The results indicate that the average performance of the algorithm using SA with constant temperature gives better solution and less average number of iterations.

\section{ACKNOWLEDGEMENTS}

The authors would like to thank Jordan University of Science and Technology for supporting this work.

\section{REFERENCES}

[1] G. B. Dantzig and J. H. Ramser, "The Truck Dispatching Problem," Management Science, vol. 1, no. 6, pp. 80-91, 1959, doi: 10.1287/mnsc.6.1.80.

[2] W. Cao and W. Yang, "A Survey of Vehicle Routing Problem," Proceedings of the 13th Global Congress on Manufacturing and Management (GCMM), no. 6, 2016, pp. 1-6, doi: 10.1051/matecconf/201710001006.

[3] J. Faulin, A. Juan, E. Lera and S. Grasman, "Solving the Capacitated Vehicle Routing Problem with Environmental Criteria Based on Real Estimations in Road Transportation: A Case Study," Procedia Social and Behavioral Sciences, vol. 20, no. 1, pp. 323-334, 2011, doi: 10.1016/j.sbspro.2011.08.038.

[4] B. Mahvash, S. Chauhan and A. Awasthi, "A Column Generation Based Heuristic for the Capacitated Vehicle Routing Problem with Three-Dimensional Loading Constraints," International Journal of Production Research, vol. 55, no. 6, pp. 1730-1747, 2017, doi: 10.1080/00207543.2016.1231940.

[5] A. Lim and X. Zhang, "A Two-Stage Heuristic with Ejection Pools and Generalized Ejection Chains for the Vehicle Routing Problem with Time Windows," INFORMS Journal on Computing, vol. 19, no. 3, pp. 443-457, 2007, doi: 10.1287/ijoc.1060.0186.

[6] E. Yuliza, F. M. Puspita and S. S. Supadi, "The Robust Counterpart Open Capacitated Vehicle Routing Problem with Time Windows on Waste Transport Problems," Bulletin of Electrical Engineering and Informatics (BEEI), vol. 9, no. 5, pp. 2074-2081, 2020, doi: 10.11591/eei.v9i5.2439.

[7] N. El-Sherbeny, "Vehicle Routing with Time Windows: An Overview of Exact, Heuristic and Metaheuristic Methods," Journal of King Saud University (Science), vol. 22, no. 3, pp. 123-131, 2010, doi: 10.1016/j.jksus.2010.03.002.

[8] M. Iori, J. S. González and D. Vigo, "An Exact Approach for the Vehicle Routing Problem with Two-Dimensional Loading Constraints,” Transportation Science, vol. 41, no. 2, pp. 253-264, 2007, DOI:10.1287/trsc.1060.0165.

[9] R. Baldacci, A. Mingozzi, R. Roberti and R. Walfler, "An Exact Algorithm for the Two Echolen Capacitated Vehicle Routing Problem,” Operations Research, vol. 61, pp. 298-314, 2013, doi: 10.1287/opre.1120.1153.

[10] M. M. Tavakoli and A. Sami, "Particle Swarm Optimization in Solving Capacitated Vehicle Routing Problem," Bulletin of Electrical Engineering and Informatics (BEEI), vol. 2, no. 4, pp. 252-257, 2013, doi: 10.12928/eei.v2i4.190.

[11] G. Alfarisy, W. F. Mahmudy and M. H. Natsir, "Optimizing Laying Hen Diet using Multi-Swarm Particle Swarm Optimization," TELKOMNIKA (Telecommunication, Computing, Electronics and Control), vol. 16, no. 4, pp. 1712-1723, 2018, doi: 10.12928/telkomnika.v16i4.7765.

[12] F. M. Puspita, Y. Hartono, N. Z. Syaputri, E. Yuliza and W. D. Pratiwi, "Robust Counterpart Open Capacitated Vehicle Routing (RC-OCVRP) Model in Optimization of Garbage Transportation in District Sako and Sukarami, Palembang City," International Journal of Electrical and Computer Engineering (IJECE), vol. 8, no. 6, pp. 4382-4390, 2018, doi: 10.11591/ijece.v8i6.pp4382-4390.

[13] F. M. Puspita, A. S. Simanjuntak, R. Melati and S. Octarina, "Demand robust counterpart open capacitated vehicle routing problem time windows and deadline model of garbage transportation with LINGO 13.0," International Journal of Electrical and Computer Engineering (IJECE), vol. 10, no. 6, pp. 6380-6388, 2020, doi: 10.11591/ijece.v10i6.pp6380-6388.

[14] N. Metropolis, A. W. Rosenbluth, M. N. Rosenbluth and A. H. Teller, "Equation of State Calculations by Fast Computing Machines," The Journal of Chemical Physics, vol. 21, no. 6, pp. 1087-1092, 2018, doi: 10.1063/1.1699114.

[15] S. Kirkpatrick, C. D. Gelatt and M. P. Vecchi, “Optimization by Simulated Annealing," Science, vol. 220, no. 4598, pp. 671-680, 1983, doi: 10.1126/science.220.4598.671.

[16] J. Szabo, "Comparison of Methods for Generating Initial Solution for Simulated Annealing," Central European Researchers Journal, vol. 2, no. 1, pp. 37-41, 2016.

[17] M. A. Cruz-Chávez, "Neighborhood Generation Mechanism Applied in Simulated Annealing to Job Shop Scheduling Problems," International Journal of Systems Science, vol. 46, no. 15, pp. 2673-2685, 2015, doi: 10.1080/00207721.2013.876679.

[18] H. Shakouri, K. Shojaee and M. Behnam, "Investigation on the Choice of the Initial Temperature in the Simulated Annealing: A Mushy State SA for TSP," 2009 17th Mediterranean Conference on Control and Automation, 2009, pp. 1050-1055, doi: 10.1109/MED.2009.5164685. 
[19] Z. K. A. Baizal, K. M. Lhaksmana, A. A. Rahmawati, M. Kirom and Z. Mubarok, "Travel route scheduling based on user's preferences using simulated annealing," International Journal of Electrical and Computer Engineering (IJECE), vol. 9, no. 2, pp. 1275-1287, 2019, doi: 10.11591/ijece.v9i2.pp1275-1287.

[20] M. H. Alrefaei and S. Andradóttir, "Simulated Annealing Algorithm with Constant Temperature for Discrete Stochastic Optimization," Management Sciences, vol. 45, no. 5, pp. 748-764, 1999, doi: 10.1287/mnsc.45.5.748.

[21] M. H. Alrefaei and A. Diabat, "A Simulated Annealing with Ranking and Selection for Stochastic Optimization," Advanced Materials Research, vol. 488-489, pp. 1335-1340, 2012, doi: 10.4028/www.scientific.net/AMR.488489.1335.

[22] A. K. Ariyani, W. F. Mahmudy and Y. P. Anggodo, "Hybrid Genetic Algorithms and Simulated Annealing for Multi-trip Vehicle Routing Problem with Time Windows," International Journal of Electrical and Computer Engineering (IJECE), vol. 8, no. 6, pp. 4713-4723, 2018, doi: 10.11591/ijece.v8i6.pp4713-4723.

[23] V. F. Yu, A. P. Redi, Y. A. Hidayat and O. J. Wibowo, "A Simulated Annealing Heuristic for the Hybrid Vehicle Routing Problem," Applied Soft Computing, vol. 53, pp. 119-132, 2017, doi: 10.1016/j.asoc.2016.12.027.

[24] M. H. Alrefaei and A. Diabat, "A Simulated Annealing Technique for Multi-Objective Simulation Optimization," Applied Mathematics and Computation, vol. 215, no. 8, pp. 3029-3035, 2009, doi: 10.1016/j.amc.2009.09.051.

[25] M. H. Alrefaei, A. Diabat, A. Alawneh, R. Al-Aomar and M. N. Faisal, "Simulated Annealing for Multi Objective Stochastic Optimization," International Journal of Science and Applied Information Technology (IJSAIT), vol. 2, no. 2, pp. 18-21, 2013.

[26] A. Abubaker, A. Baharum and M. H. Alrefaei, "Cooling Schedule for Multi-Objective Simulated Annealing Algorithm," AIP Proceedings, vol. 1613, no. 1, pp. 55-61, 2014, doi: 10.1063/1.4894331.

[27] A. Abubaker, A. Baharum and M. H. Alrefaei, "Automatic Clustering Using Multi-Objective Particle Swarm and Simulated Annealing," PLOS ONE, vol. 10, no. 7, 2015, Art. no. e0130995, doi: 10.1371/journal.pone.0130995.

[28] A. Abubaker, A. Baharum and M. H. Alrefaei, "Multi-Objective Particle Swarm Optimization and Simulated Annealing in Practice," Applied Mathematical Sciences, vol. 10, no. 42, pp. 2087-2103, 2016, doi: 10.12988/ams.2016.64159.

[29] A. Abubaker, A. Baharum and M. H. Alrefaei, "A pruned Pareto set for multi-objective optimization problems via particle swarm and simulated annealing," International Journal of Operational Research, vol. 35, no. 1, pp. 67-86, 2019, doi: 10.1504/IJOR.2019.099544.

[30] C. W. Tsai, C. H. Hsia, S. J. Yang, S. J. Liu and Z. Y. Fang, "Optimizing hyperparameters of deep learning in predicting bus passengers based on simulated annealing," Applied Soft Computing, vol. 88, 2020, Art. no. 106068, doi: $10.1016 /$ j.asoc.2020.106068.

[31] A. S. Ramli, M. I. M Rashid and M. A. Ahmad, "Energy Management Strategy of HEV based on Simulated Annealing," International Journal of Integrated Engineering, vol. 12, no. 2, pp. 30-37, 2020.

[32] B. Liu et al., "Prediction of rock mass parameters in the TBM tunnel based on BP neural network integrated simulated annealing algorithm," Tunnelling and Underground Space Technology, vol. 95, 2020, Art. no. 103103, doi: 10.1016/j.tust.2019.103103.

[33] A. A. Kida, A. F. L. Rivas and L. A. Gallego, "An improved simulated annealing-linear programming hybrid algorithm applied to the optimal coordination of directional overcurrent relays," Electric Power Systems Research, vol. 181, 2020, Art. no. 106197, doi: 10.1016/j.epsr.2020.106197. 